\title{
Profile of Health Care Workers in a Context of Instability: A Cross-sectional Study of Four Rural Health Zones in Eastern DR Congo (Lessons Learned)
}

Charles Mushagalusa

Ecole Regionale de Sante Publique de Bukavu

\section{Bertin Kasongo}

Ecole Regionale de Sante Publique de Bukavu

Daniel Garhalangwanamuntu MAYERI ( $\nabla$ danielgamu4@gmail.com )

Universite Catholique de Bukavu Faculte de Medecine https://orcid.org/0000-0001-9316-1038

Aimé Cikomola

Ecole Regionale de Sante Publique de Bukavu

Sammuel Makali Lwamushi

Ecole Regionale de Sante Publique de Bukavu

Amani Ngaboyeka

Ecole Regionale de sante Publique de Bukavu

Lili Chishagala

Ecole Regionale de Sante Publique de Bukavu

Albert Mwembo

Ecole de Sante Publique de l'Universite de Lubumbashi

Abdon Mukalay

Ecole de Sante Publique de l'Universite de Lubumbashi

Ghislain Balaluka Bisimwa

Ecole Regionale de Sante Publique de Bukavu

\section{Research}

Keywords: Congo, unstable health district, Healthcare center, income, socio-demographic profile, Health Care worker

Posted Date: November 6th, 2020

DOI: https://doi.org/10.21203/rs.3.rs-102516/v1 
License: (c) (i) This work is licensed under a Creative Commons Attribution 4.0 International License. Read Full License 


\section{Abstract}

Background: The crisis in human resources for health is observed worldwide, particularly in sub-Saharan Africa.

Objective: This study aims to describe the profile of staff working in rural health districts in a context of crisis.

Methods: A cross-sectional study was carried out from May 15, 2017 to May 30, 2019 on 1090 health care workers (HCW) exhaustively chosen from four health districts in Eastern Democratic Republic of Congo (Idjwi, Katana, Mulungu and Walungu). The choice of health districts was based on the crisis context. The health districts were categorized in stable, in transition and unstable. A survey questionnaire was used to collect data. The $\mathrm{Chi}^{2}$ test was used for comparison of proportions and the Kruskal-Wallis test for medians. As measures of association, we calculated the odds ratios (OR) along with their $95 \%$ confidence interval. The materiality threshold was set at $5 \%$.

Results: The age of all HCW median (Min-Max) was 46 (20-84) years and female was $32 \%$. 96\% of HCW do not receive a state salary ; $64 \%$ do not receive government allowance for risk. In the stable district $\mathrm{HCW}$ were $=<34$ years old [OR $=2.0(1.5-2,6) ; \mathrm{p}<0.001]$, the matriculated HCW [OR $=2.0(1.5-2.7) ; \mathrm{p}$ $<0.0001]$, those who benefited from national and / or provincial recruitment [OR $=3.9(2.9-5.4) ; p<0.001]$, those who benefit from continuous training $[O R=2.1(1.5-2.7) ; p<0.001]$ and those who receive the local fee -for service[OR = 5.2 (1.9-14.7); $p<0.001]$. In the unstable district, men [OR = 1.7 (1.1-2.5); $p=0.009$ ], $\mathrm{HCW}=<4$ years of seniority [OR = $2.3(1.6-3.3), \mathrm{p}<0.001]$ and lower level of education [OR = $2.1(1.5-2.9)]$; $p<0.001]$. The percent of HCW who has monthly income $>=\$ 151$ is $21 \%$ in the stable zone, $9.2 \%$ in the intermediate zones and $0.9 \%$ in the unstable zone.

\section{Conclusion:}

DR Congo authorities should develop incentive mechanisms to motivate young and trained HCW to work in unstable and intermediate health districts by improving their living and working conditions.

\section{Background}

The health and well-being of the world's populations are closely linked to the performance of their health systems [1]. This performance in turn depends largely on sufficient, high-quality and motivated human resources. These are essential for providing users with quality health care that meets their individual and collective health status [2, 3]. Therefore, adequate human resource development is an important element in terms of planning, training, management and support for the professional development of health workers at all levels of the health system $[3,4,5]$.

In its report on the World Health in 2006, the World Health Organization's (WHO) has mentioned that crisis in Human Resources for Health ( $\mathrm{HRH})$ is observed worldwide and particularly in sub-Saharan Africa [3]. 
This crisis is mainly characterized by staffing problem, training profile, supervision and motivation and non-standard working conditions as well $[3,5,6,7]$. Developed countries also variously face problems of health workforce supply. In France, the number of health professionals in training is regulated by the health system. However, the existence of a plethora or shortage is rather linked to an unequal geographical distribution between urban and rural areas and a poor distribution between primary and secondary care specialties [8].

In most countries, the training of health professionals benefits from fairly rigorous regulation involving both the academic organizations and the government (Ministry of Health and Ministry of Higher Education), so as to ensure monitoring and control during their work. This makes it possible both to regulate the number of doctors and other working health professionals. It also allows assessing the training quality and ensuring respect for the technical and ethical aspects of their job. [6].

Similarly, standards and procedures for recruiting staff in health structures exist and are generally applied by the regulation bodies: either the Ministry of the Public Service, the Ministry of Planning or the Ministry of Health itself [9].

In the Democratic Republic of Congo, human resource development is one of the six axes of the strategy for strengthening the health system adopted since 2006 and revised in 2010, and a document on staff standards in health zones (districts) has been drawn up [10,11,12]. This strategy is operationalized by a health development plan. The sector diagnosis of the 1 st and 2 nd edition plans identified the main priority problems of human resources for health, in particular the imbalance in the production and inequitable distribution of Human Resources for Health, the low motivation and loyalty of health personnel, the insufficient quality of education for health professionals and the poor development of the skills of health personnel $[13,14]$. A national plan for the development of human resources for health has been drawn up in response to the problems identified. This plan aimed at «providing the health sector multidisciplinary, competent, high-performance health teams at all levels of the health pyramid, sufficient quantity and equitably distributed, contributing to the improvement of the state of health of the Congolese population through the provision of quality health care services". One of the proposed solutions is the establishment of a health information system on $\mathrm{HRH}$ and the national observatory of $\mathrm{HRH}$. The latter already exists but is not documented [15].

Inside countries in crisis such as the DRC, human resources are an essential pillar of the health system because they are already part of the system and they also allow the health system to function at its best despite the crisis context. Several authors have shown how a staff could help reduce the adverse effects of the crisis on the health system $[5,16,17,18]$. Some countries have used the crisis experience to try to reorganize their health systems. The experience of the Ebola Virus Disease (EVD) response, epidemic in Guinea, provided an opportunity to reorganize the health system by investing in the workforce. A postEbola study provided strategic guidelines for promoting the retention of health workers in rural areas (19).

As in most of African countries, the organization of the health system in the DRC is of the pyramidal type and includes three levels: the central level (National Ministry of Health), the intermediate level (Provincial 
Health Department) and the operational or peripheral level (the Health Zone) [10-14].

The DR Congo has just completed three decades of crisis and instability. Eastern DRC was the first part to be affected by the crisis with the first Rwanda war in 1994, which led to the Rwandan genocide and dumped 1 million of the refugees in the two Kivu provinces [20]. Other crisis events have followed one another (the 1998 war, the Province crisis, the 2004 crisis in South Kivu, the Kasaï Oriental crisis and various movements of insecurity observed in various regions). Thus, Eastern DRC has been considered by some authors as the region at high risk of death, with the highest mortality rate since the Second World War. This crisis, whose number of deaths was initially estimated at 3 million in 2002 [21], woke up various specialists in armed conflict situations. A second study estimated the number of deaths linked to this crisis at 5 million [22]. The country is currently considered as an "unstable country" or "fragile state", and some authors now speak of "mega crisis" [23].

Eastern DRC is still considered as a red zone and some foreign countries do not allow their citizens to visit the region despite the presence of MONUSCO for more than fifteen years $[24,25]$. The province of South Kivu is among the three most affected provinces, after North Kivu and Tanganyika. The displacement of populations due to the intensification of violent inter-community conflicts, combined with the looting of healthcare institutions, have contributed to creating a volatile situation that has led to the flight of qualified health workers from the concerned areas [23].

During these various crises, the health system was supported by both international partners and local organizations. Support from the health system was sometimes directed towards the rehabilitation of infrastructure, the supply of equipment and other inputs. This support was sometimes as subsidization of health care for the indigent and displaced populations, or direct remuneration of staff in the form of bonuses $[13,14,26]$.

At the provincial level, the Provincial Health Department (PHD) of South Kivu has grouped health zones into three categories in 2010 and 2015, according to a number of criteria: developing health zones, health zones in transition and emergency health zones. This categorization included social, economic and political conditions; insecurity or armed conflict, geographical accessibility, etc. [27].

The health institutions involved in the provision of care are either public or private, or they depend on faith-based networks. Two of these faith-based networks are predominant, namely the network of the Catholic Church through the Diocesan Office of Medical Works and the network of the Protestant Church. Although they have a monopoly on the management of resources, are registered to the National Public services and enjoy the same benefits as those in the public sector in accordance with the memorandum of understanding signed by the Ministry of Public Health [10, 14, and 27]. With the new reform of the intermediate level, six working groups have been set up within the PHD, including the human resources working group, which normally has to analyze all the problems related to HHR and propose solutions [11, 28] but up to now this commission is not operational. 
The issue of human resources only arises when people have to be assigned or decommissioned by the political authorities. Yet better control of human resources in the health system would allow decisionmakers to better plan and deploy human resources appropriately.

Then, this study aims at describing the profile of the health-workers in the 3 rural health zones in a crisis context compared to the stable health zone.

\section{Methods}

\section{Study design and population}

A cross-sectional study was carried out among 1090 healthcare workers in 128 healthcare centers in the four targeted health districts (Idjwi, Katana, Walungu and Mulungu) categorized by the Provincial Health Department into stable, transitional and unstable health districts. These four health districts were selected by convenience to represent critical cases from the other health districts.

The Idjwi health district was considered as the most stable health district in South Kivu where no displaced persons and armed gangs are observed. The Mulungu Health district (HD) is the most unstable in the province. Here internally displaced persons and crisis events are at the upper level of the province.

Intermediate districts are those that have never experienced major crises or which are post-conflict (in transition). Walungu HD is a post-conflict area and KATANA HD is an area that has never experienced major crises.

The Idjwi health district is located in Idjwi territory; the Katana health district in Kabare territory; the Mulungu health district in Shabunda territory and the Walungu health district in Walungu territory (Table 1).

The Idjwi health district is accessible by lake, the Katana and Walungu health districts are easily accessible by road, while in Mulungu health zone accessibility is difficult by road and air [29].

\section{Data collection}

An exhaustive paper survey was conducted among 1,090 health workers (medical, paramedical, administrative, and support staff) working in the four health zones. They were distributed as follows: 280 for the Idjwi HD, 364 for Katana, 165 for Mulungu and 341 for the Walungu HD. Thus, the health personnel of 128 structures, including 124 public, church and private integrated health facilities and 4 central offices of the health zones were considered in this study. The survey was conducted by PHD investigators in two stages: from May 15th to July 17th, 2017 for Katana and Walungu; from 1 April 1st to May 30th, 2019 for the Idjwi and Mulungu HDs. 
This study included all health workers, doctors, nurses, laboratory technicians, nutritionists, pharmacists, administrative and support staff working in public, church and integrated private health facilities in the health zones of Idjwi, Katana, Mulungu and Walungu who agreed to participate in our study.

Posted, laid-off or suspended health care workers were not considered in the study. The number of agents is listed in table 0 below. The health personnel surveyed all responded to our survey questionnaire.

To categorize the agents of different study areas, we used the classification of agents according to the public services of the CongoleseState which distributes agents according to the following categories: Category A (senior officials): Secretary General, Director General, Director; Category B(senior managers): Head of Division and Head of Office; Category C ( collaboration agents): 1st Class Administrative Attaché, 2nd Class Administrative Attaché and 1st Class Administrative Agent and Category D(executing agents): 2nd Class Administrative Agent, 1st Class Auxiliary Agent, 2nd Class Auxiliary Agent and the Bailiff.

\section{Data processing and analysis}

We used the Excel program for data entry. The data were then exported to Epi info 7 and SPSS 25 for processing and analysis.

The quantitative variables were described by their median following their asymmetric distributions and the qualitative variables in absolute and relative frequencies. The Chi-square test was used for the comparison of proportions and the Kruskal-Wallis test for medians.

To describe the specific characteristics of the different health zones, we made different comparisons between the zones according to the context of the crisis. As an association measure, we used the odds ratios (OR) with their $95 \%$ confidence intervals. The materiality threshold chosen is $5 \%$. 
Table 1

Characteristics of Study Health Districts

\begin{tabular}{|c|c|c|c|c|}
\hline & Health Districts & & & \\
\hline Features & Idjwi & Katana & Walungu & Mulungu \\
\hline Territory & Idjwi & Kabare & Walungu & Shabunda \\
\hline Population & 294,209 & 236,986 & 285,669 & 170,439 \\
\hline Area & 681 sqm. & 400 sqm. & 800 sqm. & 6.500 sqm. \\
\hline Density & $\begin{array}{l}432 \\
\text { inhabitants/sqm }\end{array}$ & $\begin{array}{l}592 \text { inhabitants } \\
\text { /sqm. }\end{array}$ & $\begin{array}{l}357 \\
\text { inhabitants/sqm }\end{array}$ & $\begin{array}{l}26 \\
\text { inhabitants/sqm. }\end{array}$ \\
\hline $\begin{array}{l}\text { No. of } \\
\text { Health } \\
\text { Areas }\end{array}$ & 21 & 18 & 23 & 20 \\
\hline $\begin{array}{l}\text { No. of } \\
\text { Hospitals }\end{array}$ & 4 & 2 & 3 & 2 \\
\hline $\begin{array}{l}\text { No. of } \\
\text { Agents }\end{array}$ & 280 & 364 & 165 & 341 \\
\hline $\begin{array}{l}\text { Other Fosa } \\
\text { and HC }\end{array}$ & 35 & 27 & 30 & 21 \\
\hline Manager & $\begin{array}{l}\text { Catholic faith-based } \\
\text { (BDOM) }\end{array}$ & $\begin{array}{l}\text { catholic faith-based } \\
\text { (BDOM) }\end{array}$ & State & State \\
\hline Partners & BDOM, IRC & BDOM, LC & LC & MSF, ICRC \\
\hline $\begin{array}{l}\text { Type of } \\
\text { support }\end{array}$ & Overall support & Overall support & $\begin{array}{l}\text { Strengthening the } \\
\text { Health system }\end{array}$ & $\begin{array}{l}\text { Emergency } \\
\text { support in SAs in } \\
\text { crisis }\end{array}$ \\
\hline $\begin{array}{l}\text { Socio- } \\
\text { economic } \\
\text { activities }\end{array}$ & $\begin{array}{l}\text { Agriculture, } \\
\text { Livestock and } \\
\text { artisanal fishing) }\end{array}$ & $\begin{array}{l}\text { Agriculture, artinsal } \\
\text { fishing and small } \\
\text { trade }\end{array}$ & $\begin{array}{l}\text { Agriculture, } \\
\text { livestock ad small } \\
\text { business }\end{array}$ & Agriculture \\
\hline $\begin{array}{l}\text { No. =Numb } \\
\text { Internationa } \\
\text { Center, MSF }\end{array}$ & $\begin{array}{l}\text { sqm = Square meter, } \\
\text { Rescue Committee, LC } \\
\text { Médecins Sans Fronti }\end{array}$ & $\begin{array}{l}\mathrm{OM}=\text { Bureau Diocésa } \\
\text { Louvain Cooperation, }\end{array}$ & $\begin{array}{l}\text { des Oeuvres Médica } \\
\text { OSA = Formation sal }\end{array}$ & $\begin{array}{l}\mathrm{s}, \mathrm{IRC}= \\
\text { taire, } \mathrm{HC}=\text { Health }\end{array}$ \\
\hline
\end{tabular}

\section{Results}


Table 1

(part 1). Socio-demographic characteristics of the respondents according to the Health Districts studied

\begin{tabular}{|c|c|c|c|c|c|}
\hline Variables & ALL n (\%) & $\begin{array}{l}\text { Mulungu n } \\
\text { (\%) }\end{array}$ & Idjwi n (\%) & $\begin{array}{l}\text { Katana n } \\
(\%)\end{array}$ & $\begin{array}{l}\text { Walungu n } \\
\text { (\%) }\end{array}$ \\
\hline Age (years) & $\begin{array}{l}46(20-84) \\
*\end{array}$ & $43(20-84)$ * & $\begin{array}{l}40(22-75) \\
*\end{array}$ & $\begin{array}{l}50(20-81) \\
*\end{array}$ & $49(20-81)$ * \\
\hline$\leq 34$ & $277(26)$ & $32(21)$ & $97(37)$ & $72(22 \%)$ & $76(24)$ \\
\hline$>34$ & $783(74)$ & $124(79)$ & $162(63)$ & $260(78 \%)$ & $237(76)$ \\
\hline \multicolumn{6}{|l|}{ Gender } \\
\hline Male & $737(68)$ & $120(77)$ & 192(69) & $211(64)$ & $214(68)$ \\
\hline Female & $341(32)$ & $35(23)$ & $86(31)$ & 121(36) & $99(32)$ \\
\hline \multicolumn{6}{|l|}{ Level of education } \\
\hline <superior & $601(56)$ & $66(40)$ & $88(32)$ & $215(65)$ & $232(74)$ \\
\hline Superior & $480(44)$ & $98(60)$ & 184(68) & 117(35) & $81(26)$ \\
\hline \multicolumn{6}{|l|}{ Facilities } \\
\hline HDCO & $52(5)$ & $7(5)$ & $10(4)$ & $16(5)$ & $19(6)$ \\
\hline Hospital $(\mathrm{RH}+\mathrm{HC})$ & $372(35)$ & $43(28)$ & 103(37) & $117(35)$ & $109(35)$ \\
\hline Health Center & $649(60)$ & $102(67)$ & 163(59) & $199(60)$ & 185(59) \\
\hline \multicolumn{6}{|l|}{ Statut of the facilities } \\
\hline Public & $684(65)$ & 142(99) & $144(55)$ & 192(64) & $206(60)$ \\
\hline Other & $364(35)$ & $1(1)$ & $116(45)$ & 107(36) & $140(40)$ \\
\hline \multicolumn{6}{|l|}{ Marital statut } \\
\hline Living alone & 189(17) & 28(18) & $51(17)$ & $56(17)$ & $54(17)$ \\
\hline As a couple & $919(83)$ & $129(82)$ & 255(83) & 276(83) & 259(83) \\
\hline Seniority (years) & $10(0-59)$ * & $7(0-19) *$ & $8(0-35)$ * & $13(2-59)$ * & $13(2-59)$ * \\
\hline$\leq 4$ & $289(28)$ & $61(44)$ & $90(34)$ & $73(22)$ & $65(21)$ \\
\hline$>4$ & $758(72)$ & $79(56)$ & $172(66)$ & $259(78)$ & $248(79)$ \\
\hline \multicolumn{6}{|l|}{ Family residence } \\
\hline In the working Area & $953(88)$ & 137(86) & 238(87) & $301(91)$ & $277(88)$ \\
\hline Out of working area & $126(12)$ & 22(14) & $37(13)$ & $31(9)$ & $36(12)$ \\
\hline
\end{tabular}




\begin{tabular}{|c|c|c|c|c|c|}
\hline Variables & ALL n (\%) & $\begin{array}{l}\text { Mulungu n } \\
(\%)\end{array}$ & Idjwi n (\%) & $\begin{array}{l}\text { Katana n } \\
(\%)\end{array}$ & $\begin{array}{l}\text { Walungu } \mathrm{n} \\
\text { (\%) }\end{array}$ \\
\hline Administrative officer & $571(53)$ & $84(53)$ & $130(47)$ & $168(51)$ & $189(60)$ \\
\hline $\begin{array}{l}\text { Medical Doctor and } \\
\text { nurse }\end{array}$ & $509(47)$ & $76(48)$ & $145(53)$ & 164(49) & $124(40)$ \\
\hline
\end{tabular}

Table 1 shows the socio-demographic characteristics of the population. In all health districts, subjects over 34 years old were the most represented. The median age was 46 years, the oldest health worker was 84 years old and he was from Mulungu HD. The youngest was 20 years old. Women were the less represented in all health districts especially in Mulungu (23\%). $44 \%$ of the agents had a high level of education (university or high institute). $68 \%$ the Idjwi health district had a high level of education, followed by Mulungu health district $(60 \%)$. In all health districts, the nursing/medical staff ( $47 \%)$ was less represented than the administrative staff (53\%).

$96 \%$ of health personnel in all health districts do not receive state salary and $64 \%$ do not receive the Government bonus. However, $82 \%$ of health care workers receive the local bonus and $63 \%$ of agents do not have State registration numbers. 
Table 1

(part 2). Socio-demographic characteristics of the respondents according to the Health Zones studied (continued)

\begin{tabular}{|c|c|c|c|c|c|}
\hline Variables & $\begin{array}{l}\text { ALL } n \\
(\%)\end{array}$ & $\begin{array}{l}\text { Mulungu n } \\
(\%)\end{array}$ & $\begin{array}{l}\text { Idjwin } \\
\text { (\%) }\end{array}$ & $\begin{array}{l}\text { Katana n } \\
(\%)\end{array}$ & $\begin{array}{l}\text { Walungu } n \\
(\%)\end{array}$ \\
\hline \multicolumn{6}{|l|}{ Rank* } \\
\hline Category c & $417(40)$ & $59(37)$ & $95(35)$ & 121(39) & $142(47)$ \\
\hline category d & $628(60)$ & 101(63) & $175(65)$ & $190(61)$ & $162(53)$ \\
\hline \multicolumn{6}{|l|}{ Status in Public Service } \\
\hline Registered state agent & $391(37)$ & $79(49)$ & $119(46)$ & $117(35)$ & $76(24)$ \\
\hline non -registered state agent & $675(63)$ & $82(51)$ & $141(54)$ & $215(65)$ & $237(76)$ \\
\hline \multicolumn{6}{|l|}{ Recruitment authority } \\
\hline $\begin{array}{l}\text { central and intermediate } \\
\text { level }\end{array}$ & $629(59)$ & $120(73)$ & $85(32)$ & $225(68)$ & 199(64) \\
\hline other & $442(41)$ & $44(27)$ & $177(68)$ & 107(32) & $114(36)$ \\
\hline \multicolumn{6}{|l|}{ Recruitment process } \\
\hline Job application & $491(46)$ & $18(11)$ & 158(59) & $173(52)$ & $142(45)$ \\
\hline other & $588(54)$ & 146(89) & $112(41)$ & $159(48)$ & $171(55)$ \\
\hline \multicolumn{6}{|l|}{ Continuing education } \\
\hline Have continuing education & $205(49)$ & $55(36)$ & $150(56)$ & $129(41)$ & $119(36)$ \\
\hline $\begin{array}{l}\text { Don't have continuing } \\
\text { education }\end{array}$ & $216(51)$ & $99(64)$ & $117(44)$ & 184(59) & 213(64) \\
\hline \multicolumn{6}{|l|}{ Promotion since enlistment } \\
\hline Yes & $121(28)$ & $47(29)$ & $74(27)$ & $88(28)$ & 113(34) \\
\hline No & $313(72)$ & $117(71)$ & 196(73) & $225(72)$ & $219(66)$ \\
\hline \multicolumn{6}{|l|}{ Receive state salary } \\
\hline Yes & $16(4)$ & $5(3)$ & $11(4)$ & $18(6)$ & $16(5)$ \\
\hline no & $411(96)$ & 157(97) & $254(96)$ & 295(94) & $316(95)$ \\
\hline \multicolumn{6}{|l|}{ Receive local fee for-service } \\
\hline Yes & $356(82)$ & $83(52)$ & 273(99) & $304(97)$ & 295(89) \\
\hline no & $80(18)$ & $76(48)$ & $4(1)$ & $9(3)$ & $37(11)$ \\
\hline
\end{tabular}




\begin{tabular}{|c|c|c|c|c|c|}
\hline Variables & $\begin{array}{l}\text { ALL n } \\
(\%)\end{array}$ & $\begin{array}{l}\text { Mulungu n } \\
(\%)\end{array}$ & $\begin{array}{l}\text { Idjwi n } \\
\text { (\%) }\end{array}$ & $\begin{array}{l}\text { Katana n } \\
\text { (\%) }\end{array}$ & $\begin{array}{l}\text { Walungu } n \\
\text { (\%) }\end{array}$ \\
\hline \multicolumn{6}{|c|}{ Receive the Government allowance for risk } \\
\hline Receive & $158(36)$ & $64(39)$ & $94(35)$ & $18(5)$ & 197(64) \\
\hline Don't receive & $276(64)$ & $99(61)$ & $177(65)$ & $318(95)$ & 112(36) \\
\hline
\end{tabular}


Table 2.1

Comparison of personnel in the stable health district with other health districts

Idjwi and Mulungu

$\begin{array}{llllllll}\text { N } & \text { Idjwi } & \text { OR(IC95\%) } & \text { P } & \text { N } & \text { IDJWI } & \text { OR(IC95\%) } & \text { p }\end{array}$

\section{Age (years)}

$\begin{array}{lllllllll}\leq 34 & 129 & 75 \% & 2.3(1.5-3.7) & < & 169 & 57 \% & 2.2(1.5- & < \\ >34 & 286 & 57 \% & 1 & & & 422 & 38 \% & 1\end{array}$

\section{Gender}

$\begin{array}{lllllllll}\text { Male } & 312 & 62 \% & 1 & & 403 & 48 \% & 1 & \\ \text { Female } & 121 & 71 \% & 1.5(0.9-2.4) & 0.063 & 207 & 42 \% & 0.8(0.6- & 0.152\end{array}$

\section{Level of education}

$<$ Superior

$15457 \%$

$0.7(0.5-1.1)$

$0.09430371 \%$ **

$3.8(2.7-$

5.4)

$<$

$\begin{array}{lllllll}\text { Superior } & 282 & 65 \% & 1 & 301 & 39 \% * \star & 1\end{array}$

\section{Facilities}

$\begin{array}{lcccccccc}\text { HDCO } & 17 & 59 \% & 1 & & 26 & 38 \% & 1 & \\ \text { Hospital (RH+ HC) } & 146 & 71 \% & 1.7(0.6-4.7) & 0.321 & 220 & 47 \% & \begin{array}{l}1.4(0.6- \\ 2.2)\end{array} & 0.419 \\ & & & & & & & 2.3) \\ \text { Health Center } & 265 & 62 \% & 1.12(0.4-3.0) & 0.826 & 362 & 45 \% & \begin{array}{l}1.3(0.6- \\ 2.9)\end{array} & 0.515\end{array}$

\section{Status of the facilities}

\begin{tabular}{|c|c|c|c|c|c|c|c|c|}
\hline Public & 286 & $50 \% *$ & $\begin{array}{l}117.6(16.2- \\
853.7)\end{array}$ & $<001$ & 336 & $57 \% \star \star$ & $\begin{array}{l}1.4(1.1- \\
2.0)\end{array}$ & 0.033 \\
\hline $\begin{array}{l}\text { Other(Private,Faith- } \\
\text { based) }\end{array}$ & 117 & $1 \% *$ & 1 & & 223 & $48 \% \star \star$ & 1 & \\
\hline
\end{tabular}

\section{Marital status}

$<$ superior $=$ without, primary and secondary level, $\mathrm{OR}=$ Odds ratio, $95 \% \mathrm{Cl}=95 \%$ confidence interval, $\mathrm{HDCO}=$ Health Zone Central office, $\mathrm{RH}=$ Referral Hospital, $\mathrm{HC}=$ Health Center. *Percentage in Mulungu, ** Percentage in Katana. 


\begin{tabular}{|c|c|c|c|c|c|c|c|c|}
\hline \multirow[b]{2}{*}{ Living alone } & \multicolumn{4}{|c|}{ Idjwi and Mulungu } & \multicolumn{4}{|c|}{ Idjwi and Katana } \\
\hline & 79 & $65 \%$ & $0.9(0.6-1.5)$ & 0.752 & 107 & $48 \%$ & $\begin{array}{l}0.9(0.7- \\
1.5)\end{array}$ & 0.945 \\
\hline As a couple & 384 & $66 \%$ & 1 & & 531 & $48 \%$ & 1 & \\
\hline \multicolumn{9}{|l|}{ Seniority } \\
\hline$<5$ years & 151 & $60 \%$ & $0.7(0.4-1.0)$ & 0.068 & 163 & $55 \%$ & $\begin{array}{l}1.9(1.3- \\
2.7)\end{array}$ & <. \\
\hline$>=5$ years & 251 & $69 \%$ & 1 & & 431 & $40 \%$ & 1 & \\
\hline \multicolumn{9}{|l|}{ Family residence } \\
\hline In the work Area & 375 & $63 \%$ & 1 & & 539 & $44 \%$ & 1 & \\
\hline Out of working area & 59 & $63 \%$ & $0.9(0.6-1.7)$ & 0.910 & 68 & $54 \%$ & $\begin{array}{l}1.5(0.9- \\
2.5)\end{array}$ & 0.109 \\
\hline \multicolumn{9}{|c|}{ Position in the Facilities } \\
\hline $\begin{array}{l}\text { Administrative } \\
\text { officer }\end{array}$ & 214 & $61 \%$ & 1 & & 298 & $44 \%$ & 1 & \\
\hline $\begin{array}{l}\text { Medical Doctor and } \\
\text { nurse }\end{array}$ & 221 & $66 \%$ & $1.2(0.8-1.8)$ & 0.292 & 309 & $47 \%$ & $\begin{array}{l}1.1(0.8- \\
1.6)\end{array}$ & 0.414 \\
\hline $\begin{array}{l}<\text { superior }=\text { without, } p \\
\text { HDCO = Health Zone } \\
\text { Mulungu, ** Percenta }\end{array}$ & $\begin{array}{l}\text { imary } \\
\text { entral }\end{array}$ & $\begin{array}{l}\text { nd sec } \\
\text { ffice, } F\end{array}$ & $\begin{array}{l}\text { dary level, OR = } \\
=\text { Referral Hosp }\end{array}$ & $\begin{array}{l}\text { dds rat } \\
\text { al, } \mathrm{HC}=\end{array}$ & $95 \%$ & $\begin{array}{l}I=95^{\circ} \\
\text { enter. }\end{array}$ & $\begin{array}{l}\text { onfidence i } \\
\text { rrcentage il }\end{array}$ & \\
\hline
\end{tabular}


Table 2.2

Comparison of personnel in the stable health zone (Idjwi) with other health zones Idjwi and Walungu

Idjwi and (Katana-Walungu)

$\begin{array}{lllllllll}\text { Variables } & n & \text { Idjwi } & \begin{array}{l}\text { OR } \\ \text { (IC95\%) }\end{array} & \text { P } & \text { N } & \text { Idjwi } & \begin{array}{l}\text { OR } \\ \text { (ilC95\%) }\end{array} & P\end{array}$

Age (year)

$\begin{array}{lllllllll}\leq 34 & 173 & 56 \% & \begin{array}{l}1.9(1.3- \\ 2.7)\end{array} & 0.001 & 245 & 40 \% & 2.0(1.5- & < \\ \geq 35 & 399 & 41 \% & 1 & & & 659 & 25 \% & 1\end{array}$

\section{Gender}

\begin{tabular}{|c|c|c|c|c|c|c|c|c|}
\hline Male & 406 & $47 \%$ & 1 & & 617 & $31 \%$ & 1 & \\
\hline Female & 185 & $46 \%$ & $\begin{array}{l}0.9(0.7- \\
1.4)\end{array}$ & 0.860 & 306 & $28 \%$ & $\begin{array}{l}1.2(0.9- \\
1.6)\end{array}$ & 0.347 \\
\hline
\end{tabular}

\section{Level of education}

$<$ Superior

$32073 \%$ *

$5.9(4.2-$
$8.6)$

$<.001$

$53584 \%$ **

$4.7(3.5-$

$6.4)$

$<$

Superior

$26531 \%$ * 1

$38252 \%$ ** 1

\section{Facilities}

$\begin{array}{lccllllll}\text { HDCO } & 29 & 34 \% & 1 & & 45 & 22 \% & 1 & \\ \text { Hospital (RH+ HC) } & 212 & 49 \% & \begin{array}{l}1.8(0.8- \\ 4.0)\end{array} & 0.153 & 329 & 31 \% & \begin{array}{l}1.6(0.8- \\ 3.3)\end{array} & 0.213 \\ \text { Health Center } & 348 & 47 \% & \begin{array}{l}1.7(0.8- \\ 3.7)\end{array} & 0.200 & 547 & 30 \% & \begin{array}{l}1.5(0.7- \\ 3.1)\end{array} & 0.283 \\ & & & & & & \end{array}$

\section{Status of the facilities}

Public

$350 \quad 41 \%$

$0.8(0.6-$

1.2)

0.306

$542 \quad 27 \%$

0.8(0.6-

1.0)

Other

$256 \quad 45 \% \quad 1$

$\begin{array}{lll}363 & 32 \% & 1\end{array}$

$<$ superior $=$ without, primary and secondary level, $\mathrm{OR}=$ Odds ratio, $95 \% \mathrm{Cl}=95 \%$ confidence interval, $\mathrm{HDCO}=$ Health District Central office, $\mathrm{RH}=$ Referral Hospital, $\mathrm{HC}=$ Health Center *Percentage in Walungu, ** Percentage in (Walungu-katana) 


\section{Marital status}

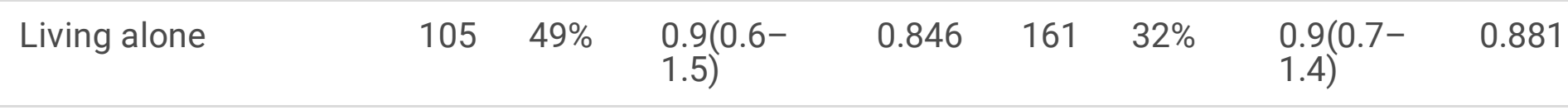

As a couple

$514 \quad 50 \% \quad 1$

$790 \quad 32 \% \quad 1$

\section{Seniority}

\begin{tabular}{|c|c|c|c|c|c|c|c|c|}
\hline$<5$ years & 155 & $58 \%$ & $\begin{array}{l}1.9(1.4- \\
2.9)\end{array}$ & $\stackrel{<}{0.001}$ & 228 & $39 \%$ & $\begin{array}{l}1.9(1.4- \\
2.6)\end{array}$ & $\begin{array}{l}< \\
0.001\end{array}$ \\
\hline$>=5$ & 420 & $41 \%$ & 1 & & 679 & $25 \%$ & 1 & \\
\hline
\end{tabular}

Family residence

$\begin{array}{lllllllll}\text { In the working Area } & 515 & 46 \% & 1 & & 816 & 29 \% & 1 & \\ \text { Out of working area } & 73 & 51 \% & \begin{array}{l}1.9(0.7- \\ 1.9)\end{array} & 0.474 & 104 & 36 \% & 1.3(0.9- & 0.179 \\ & & & & & & 2.1) & \end{array}$

\section{Position in the Facilities}

$\begin{array}{lllllllll}\text { Administrative officer } & 319 & 41 \% & 1 & & 487 & 27 \% & 1 & \\ \begin{array}{l}\text { Medical Doctor and } \\ \text { nurse }\end{array} & 269 & 54 \% & \begin{array}{l}1.7(1.2- \\ 2.4)\end{array} & 0.001 & 433 & 33 \% & \begin{array}{l}1.4(1.1- \\ 1.8)\end{array} & 0.024 \\ & & & & & & \end{array}$

<superior = without, primary and secondary level, OR = Odds ratio, $95 \% \mathrm{Cl}=95 \%$ confidence interval, $\mathrm{HDCO}=$ Health District Central office, $\mathrm{RH}=$ Referral Hospital, $\mathrm{HC}=$ Health Center *Percentage in Walungu, ** Percentage in (Walungu-katana) 
Table 2.3

Comparison of personnel in the stable health zone with other health zones (continued) Idjwi and Mulungu Idjwi and Katana

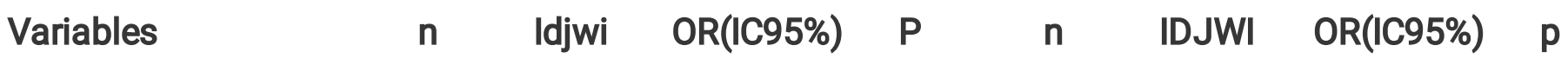

Rank****

Category C

$154 \quad 62 \% \quad 0.9(0.6-$

1.4)

$0.724 \quad 216 \quad 44 \%$

$0.9(0.6-$

0.355

category D

$276 \quad 63 \% \quad 1$

$365 \quad 48 \% \quad 1$

\section{Status in Public Service}

$\begin{array}{llllllll}\text { Registered state agent } & 198 & 60 \% & \begin{array}{l}0.9(0.6- \\ 1.3)\end{array}\end{array} \quad \begin{array}{llll}0.510 & 236 & 50 \% & \begin{array}{l}1.6(1.1- \\ 2.2)\end{array}\end{array} \quad 0.009$

$\begin{array}{lllllll}\text { non-registered agent } & 223 & 63 \% & 1 & 356 & 40 \% & 1\end{array}$

\section{Recruitment authority}

central and

intermediate level

peripheral level and

Faith-based
$20559 \% *$

8.7)

$22120 \% * 1$
$5.7(3.7-$ < $31073 \%$ **

0.001

$31073 \% \star \star$

4.4(3.1-

$6.2)$

$28438 \%$ ** 1

\section{Recruitment process}

Job application

Other***

\section{Continuing education}

Yes

No

\section{Promotion since enlistment}

$33148 \%$

0.001

19.8)

$258 \quad 43 \% \quad 1$

$\begin{array}{lllll}< & 279 & 54 \% & \begin{array}{l}1.8(1.3- \\ 2.5)\end{array} & <.001 \\ 0.001 & & & 0.001 \\ & 301 & 39 \% & 1 & \end{array}$

$<$ 0.001

1




\begin{tabular}{|c|c|c|c|c|c|c|c|c|}
\hline \multirow[b]{2}{*}{ yes } & \multicolumn{4}{|c|}{ Idjwi and Mulungu } & \multicolumn{4}{|c|}{ Idjwi and Katana } \\
\hline & 121 & $61 \%$ & $\begin{array}{l}0.9(0.6- \\
1.5)\end{array}$ & 0.778 & 162 & $46 \%$ & $\begin{array}{l}0.9(0.7- \\
1.4)\end{array}$ & 0.849 \\
\hline No & 313 & $63 \%$ & 1 & & 421 & $47 \%$ & 1 & \\
\hline \multicolumn{9}{|c|}{ Receive state salary } \\
\hline Yes & 16 & $69 \%$ & $\begin{array}{l}1.4(0.5- \\
3.9)\end{array}$ & 0.574 & 29 & $38 \%$ & $\begin{array}{l}0.7(0.3- \\
1.5)\end{array}$ & 0.379 \\
\hline No & 411 & $62 \%$ & 1 & & 549 & $46 \%$ & 1 & \\
\hline \multicolumn{9}{|c|}{ Receive local fee for-service } \\
\hline Yes & 356 & $77 \%$ & $\begin{array}{l}62.5(22.2- \\
175.9)\end{array}$ & $<.001$ & 577 & $47 \%$ & $\begin{array}{l}2.1(0.6- \\
6.6)\end{array}$ & 0.237 \\
\hline No & 80 & $5 \%$ & 1 & & 13 & $31 \%$ & 1 & \\
\hline \multicolumn{9}{|c|}{ Receive the government allowance for risk } \\
\hline ou & 158 & $59 \%$ & $\begin{array}{l}0.8(0.6- \\
1.2)\end{array}$ & 0.337 & 112 & $84 \%$ & $\begin{array}{l}9.4(5.5- \\
16.1)\end{array}$ & $\begin{array}{l}<.001 \\
0.001\end{array}$ \\
\hline non & 276 & $64 \%$ & 1 & & 495 & $36 \%$ & 1 & \\
\hline \multicolumn{9}{|c|}{$\begin{array}{l}\text { OR = Odds ratio, IC } 95 \%=95 \% \mathrm{Cl}=95 \% \text { confidence interval, * Percentage in Mulungu, } * * \text { Percentage in } \\
\text { katana } * \star * \text { Other }=\text { Integration after internship and higher instances recommendation, } * * \star * \text { according } \\
\text { to the categorization of agents according to the public service of the DRC (explanation in the } \\
\text { methodology), }\end{array}$} \\
\hline
\end{tabular}


Table 2.4

Comparison of the different characteristics of personnel in the stable health zone (Idjwi) with other health zones, intermediate (Walungu and Katana) and in crisis (Mulungu) according to the emergency classification of the provincial health department (DP) from South Kivu, East of the DRC (continued)

\begin{tabular}{|c|c|c|c|c|c|c|c|c|}
\hline \multirow[b]{2}{*}{ Variables } & \multicolumn{4}{|c|}{ Idjwi and Walungu } & \multicolumn{4}{|c|}{ Idjwi and (Katana-Walungu) } \\
\hline & $\mathrm{n}$ & Idjwi & $\begin{array}{l}\text { OR } \\
\text { (ic95\%) }\end{array}$ & $\mathbf{p}$ & $\mathbf{N}$ & Idjwi & $\begin{array}{l}\text { OR } \\
\text { (ic95\%) }\end{array}$ & $\mathbf{p}$ \\
\hline \multicolumn{9}{|l|}{$\operatorname{Rank}^{\star \star \star \star}$} \\
\hline Category C & 237 & $60 \% *$ & $\begin{array}{l}1.6(1.2- \\
2.3)\end{array}$ & 0.005 & 358 & $73 \% * \star$ & $\begin{array}{l}1.4(1.1- \\
1.9)\end{array}$ & 0.032 \\
\hline Category D & 337 & $48 \% *$ & 1 & & 526 & $67 \%$ ** & 1 & \\
\hline \multicolumn{9}{|l|}{ Status in Public Service } \\
\hline Registered state agent & 195 & $61 \%$ & $\begin{array}{l}2.6(1.8- \\
3.8)\end{array}$ & $<.001$ & 312 & $38 \%$ & $\begin{array}{l}2.0(1.5- \\
2.7)\end{array}$ & $\begin{array}{l}< \\
0.001\end{array}$ \\
\hline Non-registered agent & 378 & $37 \%$ & 1 & & 593 & $24 \%$ & 1 & \\
\hline \multicolumn{9}{|l|}{ Recruitment authority } \\
\hline $\begin{array}{l}\text { central and intermediate } \\
\text { level }\end{array}$ & 284 & $70 \% *$ & $\begin{array}{l}3.6(2.6- \\
5.1)\end{array}$ & $<.001$ & 509 & $83 \% \star \star$ & $\begin{array}{l}3.9(2.9- \\
5.4)\end{array}$ & $<.001$ \\
\hline $\begin{array}{l}\text { peripheral level- } \\
\text { confessional }\end{array}$ & 291 & $39 \% *$ & 1 & & 398 & $56 \%$ ** & 1 & \\
\hline \multicolumn{9}{|l|}{ Recruitment process } \\
\hline Job application & 300 & $53 \%$ & $\begin{array}{l}1.7(1.2- \\
2.36)\end{array}$ & 0.001 & 473 & $33 \%$ & $\begin{array}{l}1.5(1.1- \\
1.9)\end{array}$ & 0.008 \\
\hline Other*** & 283 & $40 \%$ & 1 & & 442 & $25 \%$ & 1 & \\
\hline \multicolumn{9}{|l|}{ Continuing education } \\
\hline Yes & 269 & $56 \%$ & $\begin{array}{l}2.3(1.7- \\
3.2)\end{array}$ & $<.001$ & 398 & $38 \%$ & $\begin{array}{l}2.1(1.5- \\
2.7)\end{array}$ & $\begin{array}{l}<.001 \\
0.001\end{array}$ \\
\hline No & 330 & $35 \%$ & 1 & & 514 & $23 \%$ & 1 & \\
\hline \multicolumn{9}{|l|}{$\begin{array}{l}\text { Promotion since } \\
\text { enlistment }\end{array}$} \\
\hline Yes & 187 & $40 \%$ & $\begin{array}{l}0.7(0.5- \\
1.0)\end{array}$ & 0.080 & 275 & $27 \%$ & $\begin{array}{l}0.8(0.6- \\
1.1)\end{array}$ & 0.258 \\
\hline
\end{tabular}

OR $=$ Odds ratio, $95 \% \mathrm{Cl}=95 \%$ confidence interval, *Percentage in Walungu, $* *$ Percentage in

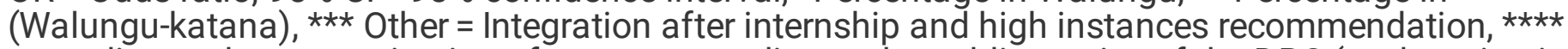
according to the categorization of agents according to the public service of the DRC (explanation in the methodology), 


\begin{tabular}{|c|c|c|c|c|c|c|c|c|}
\hline \multirow[b]{2}{*}{ No } & \multicolumn{4}{|c|}{ Idjwi and Walungu } & \multicolumn{4}{|c|}{ Idjwi and (Katana-Walungu) } \\
\hline & 415 & $47 \%$ & 1 & & 640 & $31 \%$ & 1 & \\
\hline \multicolumn{9}{|l|}{ Receive state salary } \\
\hline Yes & 27 & $41 \%$ & $\begin{array}{l}0.8(0.4- \\
1.9)\end{array}$ & 0.696 & 45 & $24 \%$ & $\begin{array}{l}0.8(0.4- \\
1.6)\end{array}$ & 0.479 \\
\hline No & 570 & $45 \%$ & 1 & & 865 & $29 \%$ & 1 & \\
\hline $\begin{array}{l}\text { Receive local fee for- } \\
\text { service }\end{array}$ & & & & & & & 1 & \\
\hline Yes & 568 & $48 \%$ & $\begin{array}{l}8,6(3,0- \\
24,3)\end{array}$ & $<0,001$ & 872 & $31 \%$ & $\begin{array}{l}5,2(1,9- \\
14,7)\end{array}$ & $<0,001$ \\
\hline No & 41 & $10 \%$ & 1 & & 50 & $8 \%$ & & \\
\hline \multicolumn{9}{|c|}{$\begin{array}{l}\text { Receive the government } \\
\text { allowance for risk }\end{array}$} \\
\hline Yes & 291 & $68 \% *$ & $\begin{array}{l}3,3(2,4- \\
4,7)\end{array}$ & $<0$ & 309 & $30 \%$ & $\begin{array}{l}1,1(0,8 \\
-1,4)\end{array}$ & 0,693 \\
\hline No & 289 & $39 \% *$ & 1 & & 607 & $29 \%$ & 1 & \\
\hline \multicolumn{9}{|c|}{$\begin{array}{l}\mathrm{OR}=\text { Odds ratio, } 95 \% \mathrm{Cl}=95 \% \text { confidence interval, *Percentage in Walungu, } * \star \\
\text { (Walungu-katana), } \\
\text { (W** Other }=\text { Integration after internship and high instances recommendation, } \\
\text { according to the categorization of agents according to the public service of the DRC (explanation in } \\
\text { the methodology), }\end{array}$} \\
\hline
\end{tabular}

Table 2 shows comparison of the different characteristics of personnel from the stable health district (Idjwi) to other health districts, intermediate (Walungu and Katana) and in crisis (Mulungu).

Health care workers $\leq 34$ years old were 2.3 times more likely to be in the Idjwi HD (than in the Mulungu $\mathrm{HD}$ ) $[\mathrm{OR}=2.3(1.5-3.7) ; \mathrm{p}<0.001], 2.2$ times more likely to be in the Idjwi HD (than in the Katana HD) [OR $=2.2(1.5-3.1) ; p<0.0001]$ and 1.9 times more likely to be in the Idjwi HD (than in the Walungu HD) compared to $>34$ years old $[\mathrm{OR}=1.9(1.3-2.7) ; \mathrm{p}<0.001$.

The < superior level of education were 3.8 times more likely to be in the Katana HD (than in the Idjwi HD) [OR = $3.8(2.7-5.4) ; p<0.0001]$ and 5.9 times more likely to be in the Walungu HD (than in the Idjwi HD) compared to the superior $[\mathrm{OR}=5.9(4.2-8.6) ; \mathrm{p}<0.001]$. (Table 2.2).

Public status was 1.4 times more likely to be in the Katana health HD (than in the Idjwi HD) compared to other status. [OR $=1.4(1.1-2.0), 0.033]$. (Table 2.2).

Doctor \& Nurse were 1.7 times more likely to be in Idjwi HD (than in Walungu HD) [OR $=1.7(1.2-2.4) ; p<$ 0.01 ] and 1.4 times more likely to be in Idjwi HD (than in Walungu HD) [OR $=1.4(1.1-1.8), p=0.024]$.

(Table 2.2). 
The $<5$ years seniority were 1.9 times more likely to be in the Idjwi HD (than in the Katana HD) [OR $=1.9$ (1.3-2.7); $p<0.001$ ] and 1.9 times more likely to be in the Idjwi HD (than in the Walungu HD) compared to $>=5$ years $[\mathrm{OR}=1.9(1.4-2.9) ; \mathrm{p}<0.001]$.

Category $\mathrm{C}$ were 1.6 times more likely to be in Walungu HD (than in Idjwi HD) compared to Category $D$ [OR $=1.6(1.2-2.3) ; p=0.005]$. (Table 2.4)

The registered agent were 1.6 times more likely to be in the Idjwi HD (than in the Katana HD) [OR $=1.6$ $(1.1-2.2) ; p=0.009$ ] (Table 2.3) and 2.6 times more likely to be in the Idjwi HD (than in the Walungu HD) compared to the other agents [OR $=2.6(1.8-3.8) ; \mathrm{p}<0.001]$. (Table 2.4).

The central and intermediate recruitment were 5.7 times more likely to be in the Mulungu HD (than in the Idjwi HD) [OR = 5.7 (3.7-8.7); $p<0.0001]$ (Table 2.3), 4.4 times more likely to be in the Katana HD (than in the Idjwi HD) $[O R=4.4(3.1-6.2) ; p<0.001]$ and 3.6 times more likely to be in Walungu HD (than in Idjwi $H D)$ compared to other agents [OR $=3.6(2.6-5.1) ; p<0.001]$.

Recruitment applications were 11.4 times more likely to be in the Mulungu HD (than in the Idjwi HD) [OR $=11.4(6.6-19.8) ; \mathrm{p}<0.001$ ] (Table 2.3) and 1.7 times more likely to be in the Walungu HD (than in the Idjwi HD) compared to the other agents [OR $=1.7(1,2-2,36) ; p<0.001]$.

Continuing education was 2.3 times more likely to be in the Idjwi HD (than in the Mulungu HD) [OR $=2.3$ (1.5-3.5); $p<0.001], 1.8$ times more likely to be in the Idjwi HD (than in the Katana HD [OR = 1.8 (1.3-2.5); $p<0.001$ ] (Table 2.3) and 2.3 times more likely to be in the Idjwi HD (than in the Walungu HD) compared to others $[\mathrm{OR}=2.3(1.7-3.2) ; \mathrm{p}<0.001]$. (Table 2.4$)$

By comparing the stable district (Idjwi HD) and the intermediate districts (Walungu and Katana HD), $\leq 34-$ year old were twice as likely to be in the Idjwi health district (as in the inner Walungu and katana districts) compared to $>34$ years old [OR $=2.0(1.5-2,6) ; p<0.001]$, (Table 2.2) The < superior level of education were 4.7 times more likely to be in the intermediate health districts (than in the Idjwi health district) compared to the superior $[\mathrm{OR}=4.7(3.5-6,4) ; \mathrm{p}<0.001]$, The $<5$ years seniority were 1.9 times more likely to be in the Idjwi Health district (than in the Intermediate HD) compared to > 5 years $[1.9(1.4-2.6) ; p<$ 0.001], Category $C$ were 1.4 times more likely to be in intermediate HD (than in Idjwi HD) compared to Category $D$ [OR $=1.4(1.1-1.9) ; p=0.032]$, the registered agent were 2.0 times more likely to be in the Idjwi $\mathrm{HD}$ (than in the intermediate HD) compared to the other agents [OR $=2.0(1.5-2.7) ; p<0.0001]$, (Table 2.4) the central \& intermediate recruitments were 3.9 times more likely to be in the Idjwi HD compared to the other agents [OR $=3.9(2.9-5.4) ; p<0.001]$, the recruiting applications were 1.5 times more likely to be in the Idjwi HD compared to the other agents [OR $=1.5(1.1-1.9) ; p=0.008]$, the continuing education were 2.1 times more likely to be in the Idjwi $\mathrm{HD}$ (than in the Intermediary HDs) compared to others [OR $=2.1(1.5-2.7) ; p<0.001]$, receiving the local Bonus were 5.2 times more likely to be in the Idjwi HD (than in the Intermediate HDs) compared to others [OR = $5.2(1.9-14.7) ; p<0.001]$ 
Table 3

Comparison of the different characteristics of personnel from the unstable health zone (Mulungu) to the transition health zones (Walungu and Katana) on the one hand and of all the other zones on the other hand (stable and intermediate), in the province of South Kivu, East of the DRC

\begin{tabular}{|c|c|c|c|c|c|c|c|c|}
\hline \multirow[b]{2}{*}{ Variables } & \multicolumn{4}{|c|}{$\begin{array}{l}\text { Comparison between unstable and } \\
\text { intermediate zones }\end{array}$} & \multicolumn{4}{|c|}{$\begin{array}{l}\text { Comparaison between unstable zones } \\
\text { and stable / intermediate zones }\end{array}$} \\
\hline & $\mathbf{N}$ & Instable & OR(IC95\%) & $p$ & $\mathbf{N}$ & Instable & OR(IC95\%) & $\mathbf{P}$ \\
\hline \multicolumn{9}{|l|}{ Age (year) } \\
\hline$\leq 34$ & 180 & $18 \%$ & $\begin{array}{l}1.2(0.8- \\
1.8)\end{array}$ & 0.514 & 277 & $12 \%$ & $1.4(0.9-2.2)$ & 0.083 \\
\hline$>34$ & 621 & $20 \%$ & 1 & & 783 & $16 \%$ & & \\
\hline \multicolumn{9}{|l|}{ Gender } \\
\hline Male & 545 & $22 \%$ & $\begin{array}{l}1.8(1.8- \\
2.7)\end{array}$ & 0.006 & 737 & $16 \%$ & $1.7(1.1-2.5)$ & 0.009 \\
\hline Female & 255 & $14 \%$ & 1 & & 341 & $10 \%$ & & \\
\hline \multicolumn{9}{|l|}{$\begin{array}{l}\text { Level of } \\
\text { education }\end{array}$} \\
\hline <superior & 513 & $87 \% *$ & $\begin{array}{l}3.4(2.3- \\
4.8)\end{array}$ & $\begin{array}{l}< \\
0.001\end{array}$ & 601 & $89 \% \star \star$ & $2.1(1.5-2.9)$ & $\begin{array}{l}<.001 \\
0.001\end{array}$ \\
\hline Superior & 296 & $67 \% *$ & 1 & & 480 & $80 \%$ ** & 1 & \\
\hline \multicolumn{9}{|l|}{ Facilities } \\
\hline HDCO & 42 & $17 \%$ & 1 & & 52 & $13 \%$ & & \\
\hline $\begin{array}{l}\text { Hospital (RH+ } \\
\mathrm{HC})\end{array}$ & 269 & $16 \%$ & $\begin{array}{l}0.9(0.4- \\
2.3)\end{array}$ & 0.911 & 372 & $12 \%$ & $0.8(0.4-1.9)$ & 0.690 \\
\hline Health Center & 486 & $21 \%$ & $\begin{array}{l}1.3(0.6- \\
3.1)\end{array}$ & 0.506 & 649 & $16 \%$ & $1.2(0.5-2.7)$ & 0.666 \\
\hline \multicolumn{9}{|l|}{$\begin{array}{l}\text { Status of the } \\
\text { facilities }\end{array}$} \\
\hline Public & 540 & $26 \%$ & $\begin{array}{l}88.1(12.3- \\
634.0)\end{array}$ & $<0.001$ & 684 & $21 \%$ & $\begin{array}{l}95.1(13.2- \\
682.9)\end{array}$ & $\begin{array}{l}<.001 \\
0.001\end{array}$ \\
\hline other & 248 & $0 \%$ & 1 & & 364 & $0 \%$ & & \\
\hline \multicolumn{9}{|l|}{ Marital status } \\
\hline Living alone & 138 & $20 \%$ & $\begin{array}{l}1.1(0,7- \\
1,7)\end{array}$ & 0.816 & 189 & $15 \%$ & $1.1(0.7-1.7)$ & 0.780 \\
\hline As a couple & 664 & $19 \%$ & 1 & & 919 & $14 \%$ & & \\
\hline
\end{tabular}




\begin{tabular}{|c|c|c|c|c|c|c|c|c|}
\hline \multirow[b]{2}{*}{$<=4$ years } & \multicolumn{4}{|c|}{$\begin{array}{l}\text { Comparison between unstable and } \\
\text { intermediate zones }\end{array}$} & \multicolumn{4}{|c|}{$\begin{array}{l}\text { Comparaison between unstable zones } \\
\text { and stable / intermediate zones }\end{array}$} \\
\hline & 199 & $31 \%$ & $\begin{array}{l}2.8(1.9- \\
4.2)\end{array}$ & $<.001$ & 289 & $21 \%$ & $2.3(1.6-3.3)$ & $\begin{array}{l}< \\
0.001\end{array}$ \\
\hline$>4$ years & 586 & $13 \%$ & 1 & & 758 & $10 \%$ & & \\
\hline \multicolumn{9}{|l|}{$\begin{array}{l}\text { Family } \\
\text { residence }\end{array}$} \\
\hline $\begin{array}{l}\text { In the working } \\
\text { Area }\end{array}$ & 715 & $19 \%$ & 1 & & 953 & $14 \%$ & & \\
\hline $\begin{array}{l}\text { Out of working } \\
\text { area }\end{array}$ & 89 & $25 \%$ & $\begin{array}{l}1.4(0.8- \\
2.3)\end{array}$ & 0.214 & 126 & $17 \%$ & $1.3(0.8-2.1)$ & 0.359 \\
\hline \multicolumn{9}{|l|}{$\begin{array}{l}\text { Position in the } \\
\text { Facilities }\end{array}$} \\
\hline $\begin{array}{l}\text { Administrative } \\
\text { officer }\end{array}$ & 441 & $19 \%$ & 1 & & 571 & $15 \%$ & 1 & \\
\hline $\begin{array}{l}\text { Medical Doctor } \\
\text { and nurse }\end{array}$ & 364 & $21 \%$ & $\begin{array}{l}0.9(0.6- \\
1.3)\end{array}$ & 0.516 & 509 & $15 \%$ & $0.9(0.7-1.4)$ & 0.919 \\
\hline \multicolumn{9}{|c|}{$\begin{array}{l}\text { <superior = without, primary and secondary level, OR= Odds ratio, IC95\%=95\% Confidence Interval, } \\
\text { HDCO = Health District Central office, RH = Referral Hospital, HC = Health Center. *Percentage in } \\
\text { intermediate, **Percentage in (stable/intermediate) }\end{array}$} \\
\hline
\end{tabular}


Table 3.1

Comparison of the different characteristics of personnel from the unstable health zone (Mulungu) to the transition health zones (walungu and katana) on the one hand and of all the other zones on the other hand (stable and intermediate), in the province of South Kivu, East of the DRC (after)

\begin{tabular}{|c|c|c|c|c|c|c|c|c|}
\hline \multirow[b]{2}{*}{ Variables } & \multicolumn{4}{|c|}{$\begin{array}{l}\text { Comparison between unstable and } \\
\text { intermediate zones }\end{array}$} & \multicolumn{4}{|c|}{$\begin{array}{l}\text { Comparaison between unstable zones } \\
\text { and stable / intermediate zones }\end{array}$} \\
\hline & $\mathrm{N}$ & Instable & OR(IC95\%) & $\mathrm{p}$ & $\mathrm{N}$ & instable & OR(IC95\%) & $P$ \\
\hline \multicolumn{9}{|l|}{ Rank*** } \\
\hline Category c & 322 & $18 \%$ & $\begin{array}{l}0.8(0.5- \\
1.1)\end{array}$ & 0.173 & 417 & $14 \%$ & $\begin{array}{l}0.9(0.6- \\
1.2)\end{array}$ & 0.389 \\
\hline category d & 452 & $22 \%$ & 1 & & 627 & $16 \%$ & & \\
\hline \multicolumn{9}{|l|}{$\begin{array}{l}\text { Status in Public } \\
\text { Service }\end{array}$} \\
\hline $\begin{array}{l}\text { Registered state } \\
\text { agent }\end{array}$ & 272 & $71 \% *$ & 1 & & 391 & $80 \% * \star$ & & \\
\hline $\begin{array}{l}\text { Non- registered } \\
\text { state agent }\end{array}$ & 534 & $85 \% *$ & $\begin{array}{l}2.3(1.6- \\
3.2)\end{array}$ & $\begin{array}{l}< \\
0.001\end{array}$ & 675 & $88 \% \star \star$ & $\begin{array}{l}1.8(1.3- \\
2.6)\end{array}$ & $<0.001$ \\
\hline \multicolumn{9}{|l|}{$\begin{array}{l}\text { Recruitment } \\
\text { authority }\end{array}$} \\
\hline $\begin{array}{l}\text { central and } \\
\text { intermediate } \\
\text { level }\end{array}$ & 544 & $22 \%$ & $\begin{array}{l}1.4(0.9- \\
2.1)\end{array}$ & 0.070 & 629 & $19 \%$ & $\begin{array}{l}2.1(1.5- \\
3.1)\end{array}$ & $<$ \\
\hline other & 265 & $17 \%$ & 1 & & 442 & $10 \%$ & & \\
\hline \multicolumn{9}{|l|}{$\begin{array}{l}\text { Recruitment } \\
\text { process }\end{array}$} \\
\hline Job application & 333 & $95 \% *$ & $\begin{array}{l}7.7(4.6- \\
12.9)\end{array}$ & $<.001$ & 491 & $96 \% * \star$ & $\begin{array}{l}8.7(5.2- \\
14.4)\end{array}$ & $<0.001$ \\
\hline other & 476 & $69 \% *$ & 1 & & 588 & $75 \% * \star$ & & \\
\hline \multicolumn{9}{|l|}{$\begin{array}{l}\text { Continuing } \\
\text { education }\end{array}$} \\
\hline Yes & 303 & $18 \%$ & $\begin{array}{l}0.9(0.6- \\
1.3)\end{array}$ & 0.530 & 453 & $12 \%$ & $\begin{array}{l}0.7(0.5- \\
1.0)\end{array}$ & 0.660 \\
\hline No & 496 & $20 \%$ & 1 & & 613 & $16 \%$ & & \\
\hline \multicolumn{9}{|l|}{$\begin{array}{l}\text { Promotion since } \\
\text { enlistment }\end{array}$} \\
\hline Yes & 248 & $19 \%$ & $\begin{array}{l}0.9(0.6- \\
1.3)\end{array}$ & 0,530 & 322 & $15 \%$ & $\begin{array}{l}0.9(0.7- \\
1.3)\end{array}$ & 0.719 \\
\hline No & 561 & $21 \%$ & 1 & & 757 & $15 \%$ & & \\
\hline
\end{tabular}




\section{Receive state}

salary

\begin{tabular}{lllllllll} 
Yes & 39 & $13 \%$ & $\begin{array}{l}0.6(0.2- \\
1.5)\end{array}$ & 0.246 & 50 & $10 \%$ & $\begin{array}{l}0.6(0.2- \\
1.6)\end{array}$ & 0.301 \\
\hline No & 768 & $20 \%$ & 1 & & & 1022 & $15 \%$ &
\end{tabular}

Receive local

fee for service

\begin{tabular}{|c|c|c|c|c|c|c|c|c|}
\hline Yes & 682 & $88 \% *$ & $\begin{array}{l}13.0(8.4- \\
20.2)\end{array}$ & $<.001$ & 955 & $91 \%$ ** & $\begin{array}{l}15.9(10.5- \\
24.4)\end{array}$ & $\begin{array}{l}< \\
0.001\end{array}$ \\
\hline No & 122 & $38 \% *$ & 1 & & 126 & $40 \%$ ** & & \\
\hline
\end{tabular}

\section{Receive the government allowance for risk}

\begin{tabular}{lllllllll} 
Yes & 279 & $23 \%$ & $\begin{array}{l}1.3(0.9- \\
1.8)\end{array}$ & 0.155 & 373 & $17 \%$ & $\begin{array}{l}1.8(0.9- \\
1.8)\end{array}$ & 0.171 \\
\hline No & 529 & $19 \%$ & 1 & & & & &
\end{tabular}

OR=Odds ratio, $95 \% \mathrm{Cl}=95 \%$ Confidence Interval, *Percentage in Intermediate, **Percentage in (stable / intermediate), ${ }^{* \star}$ according to the categorization of agents according to the public service of the DRC (explanation in the methodology),

Tables 3 shows the comparison of the different characteristics of personnel from the unstable health district (Mulungu) to the transition health districts (Walungu and Katana) on the one hand and from all the other districts on the other hand (stable and intermediate), in the province of South Kivu.

These tables show on the one hand, compared to the unstable districts (Mulungu) and the intermediate districts (Katana and Walungu) that:

The following characteristics are more likely to be in the unstable district than in the intermediate districts: male [OR = 1.8 (1.8-2.7); $p=0.006$ ], public status of facilities [OR = 88.1 $(12.3-634.0) ; p<0.001]$ and $<=4$ years Seniority [OR $=2.8(1.9-4.2) ; p<0.001]$, $<$ superior Level of education [OR =3,4(2,3-4,8); $p<$ 0.001] (Table 3)..

The following characteristics are more likely to be in the intermediate zones than the unstable zone, Nonregistered agent [OR = $2.3(1.6-3.2) ; p<0.001]$, job application [OR = 7.7 (4.6-12.9); $p<0.001]$, receive local bonus [OR = $13.0(8.4-20.2) ; p<0.001]($ Table 3.1) 
Tables 3 \& 3.1 comparing on the other hand, the unstable district (Mulungu) to all the other districts (intermediate and stable) this study showed that:

The following characteristics are more likely to be in the unstable district than in all the other districts (intermediate and stable), male [OR = $1.7(1.1-2.5) ; p=0.009],<=4$ years Seniority [OR $=2.3(1.6-3.3), p<$ 0.001 ] and Level $<$ superior of education [OR = $2.1(1.5-2.9) ; p<0.001]$. (Table 3$)$

The following characteristics are more likely to be in other areas than in the unstable area: Non-registered state agent [OR $=1.8(1.3-2.6) ; p<0.001]$, central and intermediate level $[\mathrm{OR}=2.1(1.5-3.1), p<0.001]$, job application [OR = $8.7(5.2-14.4) ; p<0.001]$, receive local fee for-service [OR $=15.9(10.5-24.4) ; p<$ 0.001] (Table 3.1).

Table 4

Monthly income by occupation and level of education of respondents

\begin{tabular}{|llllll|}
\hline \multicolumn{7}{|c|}{ Monthly income by function held in the structure (\$) } & & \\
\hline & Median (Min - Max) & & & \\
& Mulungu & Katana & Walungu & Idjwi & P-value* \\
\hline Medical doctor & $167(55-355)$ & $217(60-779)$ & $387(83-805)$ & $273(167-1033)$ & $<0.001$ \\
\hline Administrative & $51(5-90)$ & $30(7-211)$ & $40(7-250)$ & $40(4-402)$ & 0.050 \\
\hline Nurses & $52(6-115)$ & $50(7-1000)$ & $63(10-250)$ & $70(7-183)$ & $<0.001$ \\
\hline * Kruskal-Wallis & & & & & \\
\hline
\end{tabular}

This Table 4 compares the median monthly salaries of health zone personnel in the different zones studied according to the type of agents. It revealed that overall, the median of agents' salaries is statistically different according to the type of agents $(<0.001)$. Doctors and nurses have different median wages $(<0.001)$ in the different areas studied. Doctors in Mulungu HD have the lowest median salary of all areas whereas the highest Doctor's salary is found in Walungu Health district.

\section{Graphique I: Monthly income of respondents by study area (chi-square test, $\mathrm{P}<0.001$ )}

These results in Graph I indicated that it was in the Idjwi health district (stable) where we found a high proportion $(21.20 \%)$ of health workers with a monthly income greater than or equal to $\$ 151$ compared to other health districts $(\mathrm{p}<0.001)$. In the Mulungu Health district (unstable), their monthly income was very low $(0.90 \%$ of $<\$ 151)$ versus $9.4 \%$ in the Katana health district and $9.2 \%$ in the Walungu health district (in transition).

\section{Discussion}


This study describes the profile of staff in rural health zones in South Kivu, eastern Democratic Republic of Congo. It takes into account the crisis context that has characterized this province for several decades. We conducted a cross-sectional study in four districts selected according to the crisis context following the classification of health zones by the Provincial Health Department of South Kivu. The Idjwi health district was chosen as stable, Katana and Walungu as intermediate and Mulungu as in crisis.

Our findings showed that agents over 34 years of age were the most represented. Women were the least represented in all the health districts but the lowest proportion was in the unstable district. In all districts, administrative staff was the most represented comparing to nursing and medical staff. $96 \%$ of the health staff did not receive a government salary and $64 \%$ did not receive a government allowance for risk. $63 \%$ of staff do not have a state registration number. With regard to the characteristics of the health districts, our results show that the characteristics of the agents found in the stable districts are 34 years old or younger, those with a registration number, those who have benefited from central and/or intermediate recruitment, those who receive continuous training and those who receive the local bonus. Our results also show that it is in the Idjwi health district (stable) where we have a high proportion of health workers with a higher monthly income than in the other districts. In the unstable district; men, staff with less than or equal to 4 years of seniority and those with a lower level of education are more likely to be in the unstable districts than in all the other districts,.

This study, which is one of the rare studies that addresses this issue in the context of crisis in the DRC, will be a reference for future analyses of health personnel and a standard commodity for support programs. This will also enhance decision-makers' actions in order to ensure equitable and efficient health personnel coverage despite the crisis context.

\section{Socio-demographic characteristics of the respondents according to the Health districts studied}

Our results showed some difference between Study Areas in terms of age of staff and seniority. Health care workers over 34 years of age were the most represented, especially in unstable districts . This shows the homogeneous nature of the agents in the African context where retirement policies are not applied in all countries. This shows that young people coming directly from school are not quickly enrolled in the employment system. Our data also showed that it is in unstable and/or intermediate areas where we have older people than elsewhere. As a result of the persistent armed conflicts, since young people are generally better able to leave the unstable area in search of places offering the best security conditions, older people find themselves competitive in this area. This is agreement with findings of Rohini Jonnalagadda Haar and Leonard S. Rubinstein [39] and Patrick Ilboudo G. et al [33], in the Burkina Faso Health Workforce Survey 2014, regarding the age of health care workers. According to them, the majority of workers were between 27 and 57 years old. Gautier and Wane in Chad corroborated our results and found that the majority of workers were between 31 and 45 years old [34]. 
Moreover, our data showed that agents in unstable districts, although they are the oldest, they have less years of experience (least seniority) compared to the other districts (less than 4 years). This shows that it is difficult to keep the same agents longer in high-risk areas. In the Democratic Republic of the Congo (DRC), a study conducted by Rishma M. et al. showed that the majority of agents have a median age of 6 years, and the study conducted by Patrick Ilboudo G. et al. showed that the majority of agents had about 10 years of experience [33]. However, these results are consistent with those found in Papua New Guinea, [35] where a significant proportion of agents had less than six years experience (44\%). It is also important to note that in developing countries, state institutions are very weak in terms of their capacity to manage and control their own resources.

For gender, our data showed that men are about twice as likely to be in the unstable district as women. In the African context in general, some cultures do not encourage women to work, especially in conflict areas. In the context of insecurity and instability, women are exposed to more violence and harassment than men. This may explain the low proportion of women in at-risk areas [36] .

Our study showed that it is in the unstable district that we find more agents with a lower level of education. They are about twice as likely to be in the unstable district as those with higher education. This can be explained by the fact that as an unstable district in terms of security, the selection criteria will not be the same as in stable ones. It should be noted that there are other areas where there are several private facilities or humanitarian organizations that attract the best agents because they often offer the best salaries. A study conducted in Papua New Guinea on health care workers in rural areas [34] is not in agreement with our study. This study showed that $82 \%$ of the workers interviewed achieved a higher level of education before beginning the training related to their health sector position. However, our results are close to those found by a cross-sectional study of the sources of income of frontline health workers in the Democratic Republic of Congo [37], which shows that the majority of health workers are secondary school graduates, with only $30 \%$ having a higher level of education and university. The age range was between 30 and 40 years, $90 \%$ of the staff were nurses and only $4 \%$ doctors.

The study shows that the majority of the health workers in our study area were married and this in all the health districts. Results of a study on rural health workers conducted in Papua New Guinea [34] do not differ from our results in terms of civil status, where the majority of the workers were married $(81 \%)$.

With regard to the status of the structures, it can be seen that $100 \%$ of the facilities in the Mulungu HD (unstable) are state-owned. This could be explained by the fact that private and religious individuals always choose to invest in stable, secure environments where the population can afford health care costs. Joyasuriya $\mathrm{R}$ et al.[34] in their study show that $50 \%$ of respondents worked in public facilities and $40 \%$ in church-owned facilities, compared to only about $3 \%$ in the private sector. The study shows that the majority of staff live with their respective families in all the health districts. This is explained by the fact that recruitment is done locally in most cases, regardless of the hiring authority. For Joyasuriya $\mathrm{R}$ et al. [34], almost all of the agents work in their districts. However, accommodation is provided to $68 \%$ of them and $57 \%$ lived in the same complex as the health facility. 
The results of the study reveal that $47 \%$ of the agents are administrative staff. Doctors and nurses are more in the stable district than in the intermediate one. The results of our study differ slightly from the results of the 2015 Country Profile Study on Human Resources for Health in the Democratic Republic of Congo [37] which revealed that at the national level, administrative staff represents $38.4 \%$.

The results of the study show that in relation to the status of civil servants (registered agent, New Unit, No status), registered agents have twice the risk of being in the stable district. This is because administrative procedures seem to be more respected. It is probably due to the context of insecurity leading to both the flight of agents and the use of available agents who have not yet completed administrative formalities. A qualitative study among these agents and their employers would be necessary to understand the reason why some of the morkers in transition districts do not have a state registration number.

\section{The Recruitment Process, Capacity Building and Method of Paying Staff}

The results of the study show that, compared to the authorities involved in recruiting agents, those who obtained their jobs after an application for employment are about eight times more likely to be in intermediate districts. As we can see in the unstable district, a small seniority of the agents got their job after an applying for it because of the context of instability.

As a district in transition, the intermediate districts would have a greater need for agents to ensure their stability. For the unstable district(Mulungu), $84.1 \%$ of the agents are hired per application and test. In the transitional district of Katana (district with a partner), recruitment by recommendation from higher authorities occupies a slightly higher proportion than in other districts. The study on the Human Resources for Health Country Profile for the Democratic Republic of Congo shows that in the DRC, recruitment is either focused on degrees(or diploma) and institutions which issued them or by competitive examination. Recruitment is based on qualifications for candidates who hold a diploma issued or recognized as equivalent by the National Education system and who are specially prepared for that career, as long as the number of candidates does not exceed the number of jobs available.

Recruitment is carried out on a competitive basis when the number of vacancies to be filled is less than the number of participants. In this case, only successful candidates who are ranked in order of merit may be appointed. It is within the framework of recruitment by degree (or diploma) that the recommendations are used as we showed in this study. This way of doing things tends to erase the normal recruitment process and to perpetuate familiarity, nepotism, tribalism, for being at a given position in the health sector.

The results of the study reveal that the continuous training of agents has the risk of being in the stable district twice. The Observatory for Human Resources for Health (HRH) in the DRC, in its study on the Human Resources for Health Country Profile of the Democratic Republic of Congo, states that in the DRC the Ministry of Health organizes thematic training within the framework of specific programs. These 
trainings are also organized in provinces. However, there are central trainers who supervise provincial trainers. In addition to this internal organization, the Ministry of Health uses higher and university education in the training of specialists. Our results show that this training is more beneficial than for agents working in stable areas. The accessibility and the geographical stability and security of stable areas will be factors that would promote the organization of training for agents in unstable or transitional areas as well.

For the local salary (premium or bonus), agents in the stable district are more likely to receive this premium than agents in intermediate and unstable districts. This difference with respect to the collection of the local premium could be explained by the socio-economic disruptions (wars and rural exodus) that the populations of these two areas have experienced over the past few years; these disruptions do not allow the health facilities to maximize incomes to ensure the premium for all agents. In addition, the unstable districts are mostly rural areas where the majority of the population is very poor. Demographic data show that more than $80 \%$ of the rural populations in the DRC live on less than a dollar a day and the majority of people who use traditional medicine are more observed in rural areas [38].

Our results then show that $96 \%$ of health personnel do not receive a government salary and $64 \%$ do not receive a government bonus. Our results are superior to those found by the cross-sectional study of the sources of income of frontline health workers in the Democratic Republic of Congo [37], showing that one-third of nurses received no form of government payment and only $18 \%$ who received both an occupational hazard allowance and a salary.

\section{Monthly Income of Workers by Occupation and Level of Education}

Our results show that physicians are the staff with the highest income compared to other agents. They then show that the incomes of physicians and nurses are distributed differently in the study areas $(\mathrm{p}<$ 0.001). Our data corroborate with those of Patrick Ilboudo G. et al 33, also showing a difference in median income between staff according to the position held $(p=0.0321)$, their study then shows that the median income for all health staff is 295.2 (59.4-797.4) $\$$ while for doctors it is 369 (59.4-646.2) $\$$. Maria Paola B. et al [34], also show in their study that in the DRC, the median monthly income of doctors is $\$ 785$ with a maximum of $\$ 4,815$; administrative staff has $\$ 166$ and a maximum of $\$ 1,396$ while nurses have a median income of $\$ 101$ and a maximum of $\$ 2,908$. Rishma M., et al [36], in the crosssectional study of the sources of income of frontline health workers in the Democratic Republic of Congo, also shows that in the DRC, monthly income from all sources was $\$ 85$, but the average was almost double( $\$ 165)$. The highest median monthly income was for non-clinical work outside the facility (\$119), followed by government wages (\$58). The lowest median monthly income came from per diems (\$9) and informal payments $(\$ 9)$. 
Our data show that there are more agents with more than $\$ 151(21 \%)$ in the stable district, while in the intermediate one this proportion is less than $10 \%$ and less than one percent in the unstable district. This difference can be explained by the low use of health services by the population due to the rural exodus and wars. This would explain the low income maximization in unstable or transitional district [39].

\section{Limitations of the study}

Despite its multiple advantages, some limitations are recognized. First, the study was conducted in only four of the thirty-four health districts in South Kivu province. Although not representative, the inclusion of three categories of health districts, including emergency, transitional (with and without partners), and stable, allowed us to understand this profile in the province. Second, the analysis on human resources was limited only to their use, which normally required studying production, use, and retirement as well. Thirdly, other aspects that seem to be important for human resources but not taken into account are the level of satisfaction of health workers, retention, working conditions including benefits, housing, allowances, etc., the consideration of other sources of income besides state salary, state and local bonuses, and finally the workers' assessments of security crises and their impact on the performance of their tasks. Finally, this study is a retrospective one which is subject to selection bias.

\section{Conclusion}

This study shows that unstable (emergency) health districts use older staff with less seniority than other zones; there are also more men, staff with lower levels of education and most of them do not have a State registration number. Staff in stable health districts benefits more from continuing education (capacity building) than those in unstable (crisis) health districts, and in terms of salary, they earn more, with a high monthly income.

Other studies would be more important to deepen the analysis on human resources by considering parameters not studied in this work, such as the production of human resources, the agents' level of satisfaction and their attitude towards the security crisis.

The State should develop incentive mechanisms to motivate young and trained personnel to work in unstable health areas, including improving their living conditions and working conditions.

\section{List Of Abbreviations}

DR: Democratic Republic of

DRC: Democratic Republic of Congo

BDOM: Bureau Diocésain des Fuvres Médicales 
EVD: Ebola Virus Disease

HD: Health District

$\mathrm{HRH}$ : Human Resources for Health

HDCO: Health District Central office

PHD: Provincial Health Department

IRC: international Rescue Committee

LC: Local committee

MSF: Médecins Sans Frontières

RH: Referral Hospital

HC: Health Center

WHO: World Health Organization

\section{Declarations}

Ethics approval

This study was approved by the Ethics committee of the Catholic University of Bukavu, DR Congo.

\section{Consent for publication}

Not applicable

\section{Availability of data and materials}

The datasets used and/or analyzed during the current study are available from the corresponding author on reasonable request

\section{Competing interest}

The authors declare that they have no competing interests

\section{Funding}




\section{Authors' Contributions}

CM and GBB: designed the protocol of the study, supervised the data collection and edited the final manuscript

LC, SM and AN contributed in data collection in the 4 targeted health districts

$\mathrm{DGM}, \mathrm{AM}, \mathrm{MC}, \mathrm{AN}$ and $\mathrm{BK}$ : contributed in data analysis and drafting the work

Other authors interpreted data and contributed in the enrichment of the manuscript

\section{Acknowledgements}

We are very grateful to all those persons who contributed to this work. Authors sincerely thank all the directors and health care workers of the Mulungu, Walungu, Idjwi and Katana Health districts for their collaboration during this study.

\section{References}

1. World Health Organization. World Health Report; towards a stronger health system. Geneva: WHO, 2000 .

2. Zurn P, Codjia L, Sall FL, World Health Organization. Retention of health workers in difficult areas in Senegal. Geneva: WHO, 2010.

3. World Health Organization. The World Health Report - Working Together for Health. Geneva: WHO, 2006.

4. Gallais C. Human resources for health in developing countries. Literature review; Solthis-Sciences Po. 2010;38.

5. World Health Organization. Global strategy on human resources for health: Workforce 2030.

6. Marchal B and Kegels G. Which role for Medicus Mundi Internationalis in Human Resources Development? Current critical issues in Human Resources for Health in developing countries. Department of Public Health, Institute of Tropical Medicine, Antwerp. Aug 2003.

7. Dambisya YM. A review of non-financial incentives for health worker retention in east and southern Africa. Health Systems Research Group, Department of Pharmacy, School of Health Sciences, University of Limpopo, South Africa. May 2007

8. Bourgueil $Y$, Mousquès $J$, Tajahmadi A. How to improve the geographical distribution of health professionals? Lessons from the international literature and measures adopted in France. Paris: Institut de recherche et documentation en économie de la santé. June 2006. Report n 534 
9. Gueye AK and Seck PS. Etude de l'accessibilité des populations aux soins hospitaliers au Sénégal, Plateforme des acteurs non étatiques pour le suivi de l'accord de Cotonou au Sénégal, Groupe thématique Santé, October 2009

10. Ministère de la fonction publique, secrétaire général. Stratégie de Renforcement du Système de Santé, $2^{\text {ème }}$ Edition, Kinshasa: République Démocratique du Congo, Ministère de la Santé; 2010

11. Ministry of Public Health. Compendium of standards for the functioning of a health zone. Kinshasa: Democratic Republic of Congo, Ministry of Health; 2012

12. Ministry of Public Health, General Secretariat. Plan National de Denveloppement Sanitaire, 20112015, Kinshasa: Democratic Republic of Congo, Ministry of Health; 2010

13. Ministry of Public Health, General Secretariat. Plan National de Denveloppement Sanitaire, 20162020, Kinshasa: Democratic Republic of Congo, Ministry of Health; 2016

14. Ministry of Public Health, General Secretariat. Plan National de Développement des Ressources Humaines pour la Santé 2011-2015. Democratic Republic of Congo, [consulted on 16/11/2019] Available on the Internet: minisanté-rhs.cd

15. Witter S, Falisse JB, Bertone MP et al. State-building and human resources for health in fragile and conflict-affected states: exploring the linkages. Human Resources for Health, $2015 ; 13(1): 33$ DOI 10.1186/s12960-015-0023-5

16. Bertone MP, Jowett M, Dale E, Witter S. Health financing in fragile and conflict-affected settings: What do we know,seven years on? Social Science \& Medicine 2019232 (2019) 209-219 DOI 10.1016/j.socscimed.2019.04.019

17. Woodward A, Sondorp E, Witter S, Martineau T. Health systems research in fragile and conflictaffected states: a research agenda-setting exercise Health Research Policy and Systems 2016 ;14(1):51 DOI 10.1186/s12961-016-0124-1

18. van de Pas R, Kolie D, Delamou A, van Damme W. Health workforce development and retention in Guinea: a policy analysis post-Ebola. Human Resources for Health $2019 ; 17: 63$ DOI 10.1186/s12960019-0400-6

19. Porignon D, Adequacy of District Health Systems in Critical Situations: Experiences in the Great Lakes Region of Africa [PhD Thesis in Public Health Sciences]. Université Libre de Bruxelles; 2003.

20. International Rescue Committee. « Mortality in the Democratic Republic of Congo, Results from a Nationwide Survey », Conducted September-November 2002, reported April 2003

21. International Rescue Committee. « Mortality in the Democratic Republic of Congo, An Ongoing Process ». Fifth and latest survey, covering the period from January 2006 to April 2007

22. Zarocostas J, Mega-crisis in DR Congo, World Report, Lancet (London, England). 2018 ;391(10118):297-298. DOI: 10.1016/S0140-6736(18)30137-5

23. Emery Brusset, Maria Bak, Cécile Collin et al. Joint Evaluation of Conflict Prevention and Peacebuilding in the Democratic Republic of Congo, Channel Research, Belgium, Synthesis Report June 2011 
24. Maxime Arquillière, Daniel Richard, Capucine Peignier, L'instabilité en RDC, entre conflit ouvert, corruption et interférence économique, Grenoble, January 2015

25. Mayaka MS, Le financement basé sur la performance dans un système de santé complexe : cas de la République Démocratique du Congo, Institut de Recherche Santé et Société (IRSS), [PhD thesis in Public Health Sciences], Université Catholique de Louvain, 2015

26. Provincial Ministry of Health, Gender and Humanitarian Affairs, Provincial Health Division. Plan Provincial de Développement Sanitaire du Sud-Kivu 2016-2020. Bukavu: Democratic Republic of Congo, Provincial Health Division; 2015

27. Kahindo MJB, Schirvel C, Godelet E, Wodon A, Porignon D, Bonami M, Réforme des structures intermédiaires de santé en République Démocratique du Congo, Santé Publique, 2014 ;26(6):849-858 DOI 10.3917/spub.146.0849

28. Provincial Ministry of Health, Gender and Humanitarian Affairs, Provincial Health Division. Annual Report 2017. Bukavu: Democratic Republic of Congo, Provincial Health Division; 2018.

29. National Health Information System (District Health Information System 2) Democratic Republic of Congo, Access to the site: https://www.snisrdc.com on October 22, 2017

30. Ministère du Plan et Suivi de la Mise en œuvre de la Révolution de la Modernité (MPSMRM),Ministère de la Santé Publique (MSP) and ICF International, Enquête Démographique et de Santé en République Démocratique du Congo 2013-2014. Rockville, Maryland, USA: MPSMRM, MSP and ICF International 2014.

31. Mutabunga bin LF, Chenge F, Criel B, Mukalay A, Luboya $O$ and Mundongo TH. Organisation, Supply and Demand of Quality Health Services: 18 Years of Experiences of Mutual Health Insurance in South Kivu Province/RD Congo International Journal of Multidisciplinary and Current Research, 2017

32. Ilboudo PGC, Ganaba R, Cunden N and Witter S. The results of the health personnel survey, Burkina Faso, FEMHealth, March 2014.

33. Gauthier $B$ and Wane W. Suivi des dépenses publiques à destination dans le secteur santé au Tchad: Analyse des résultats d'enquête; Development Research Group; The World Bank; 2005.

34. Jayasuriya R, Whittaker M, Halim G and Matineau T, Rural health workers and their work environmrent: the rule of inter-personal factors on job satisfaction of nurses in rural Papua NewGuinea. BMC Health Services Research, 2012 Jun 12 ;156 DOI 10.1186/1472-6963-12-156

35. Rishma Maini R, Hotchkiss DR and Borghi J. A cross-sectional study of the income sources of primary care health workers in the Democratic Republic of Congo, Human Resources for Health, 2017 ;15:17DOI 10.1186/s12960-017-0185-4

36. Ranson K, Poletti T, Bornemisza O, Sondorp E. Promoting health equity in conflict-affected fragile states. The Conflict and Health Programme. London School of Hygiene and Tropical Medicine, London 2007,pg 18

37. Bertone MP, Lurton $\mathrm{G}$ and Mutombo BP. Investigating the remuneration of health workers in the DR Congo: implications for the health workforce and the health system in a fragile setting, Health policy 
and planning, 2016 ;31(9):1143-1151 DOI 10.1093/heapol/czv131

38. Dhs M. République Démocratique du Congo Enquête Démographique et de Santé Rapport préliminaire. 2014;

39. Bertone MP, Lurton G, Mutombo PB. Investigating the remuneration of health workers in the DR Congo: implications for the health workforce and the health system in a fragile setting. Health Policy Plan. 2016;31(9):1143-1151. doi:10.1093/heapol/czv131

\section{Figures}

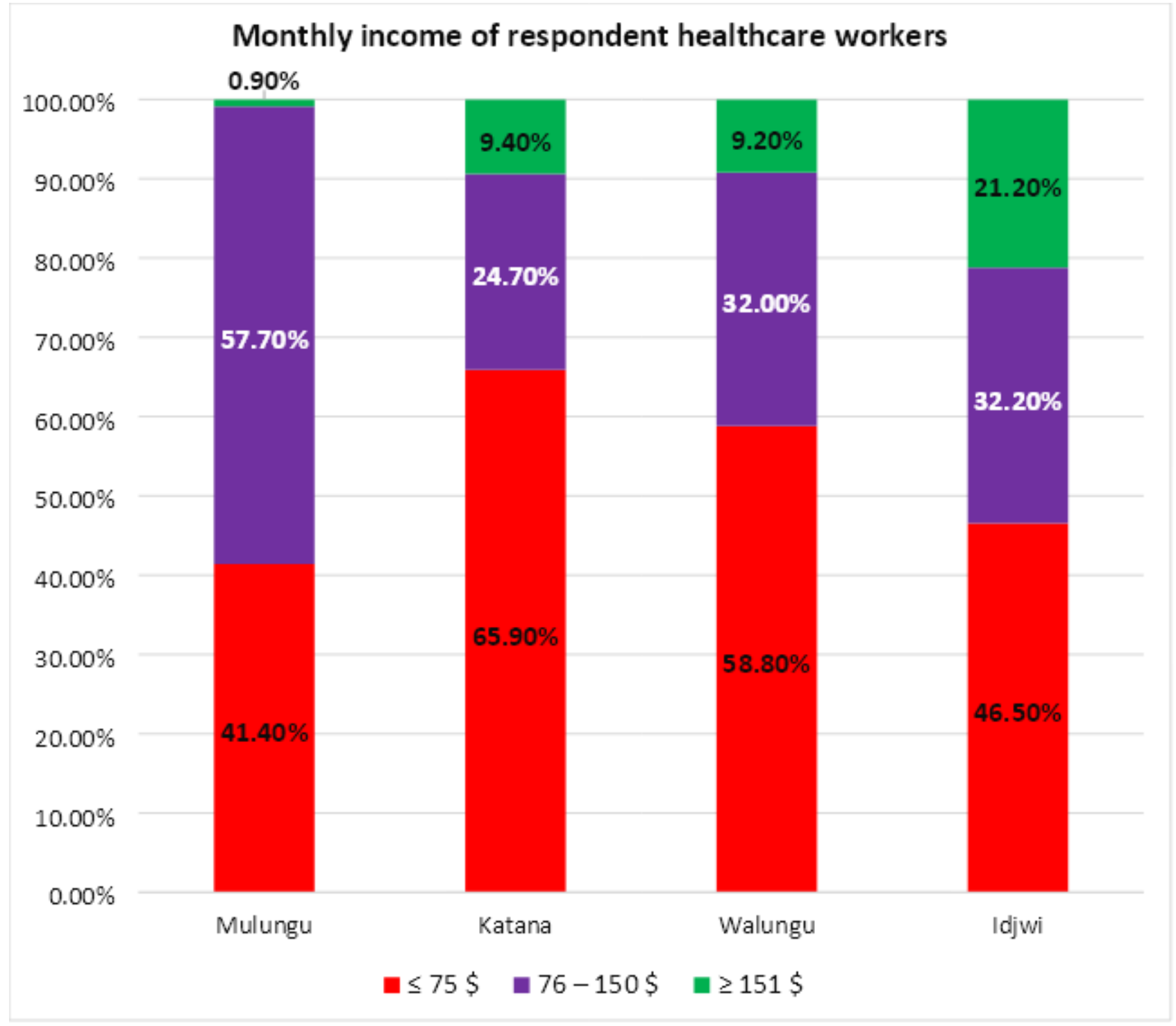

\section{Figure 1}

Graphique I: Monthly income of respondents by study area (chi-square test, $\mathrm{P}<0.001$ ) 